\title{
LiveQA: A Question Answering Dataset over Sports Live
}

\author{
Qianying Liu*12, Sicong Jiang*1, Yizhong Wang ${ }^{13}$ and Sujian Li ${ }^{1}$ \\ ${ }^{1}$ Key Laboratory of Computational Linguistics, MOE, Peking University \\ ${ }^{2}$ Graduate School of Informatics, Kyoto University \\ ${ }^{3}$ University of Washington \\ ying@nlp.ist.i.kyoto-u.ac.jp; $512580728 @ q q \cdot c o m$; \\ yizhongw@es.washington.edu; lisujian@pku.edu.cn
}

\begin{abstract}
In this paper, we introduce LiveQA, a new question answering dataset constructed from playby-play live broadcast. It contains $117 \mathrm{k}$ multiple-choice questions written by human commentators for over 1,670 NBA games, which are collected from the Chinese $\mathrm{Hupu}^{1}$ website. Derived from the characteristics of sports games, LiveQA can potentially test the reasoning ability across timeline-based live broadcasts, which is challenging compared to the existing datasets. In LiveQA, the questions require understanding the timeline, tracking events or doing mathematical computations. Our preliminary experiments show that the dataset introduces a challenging problem for question answering models, and a strong baseline model only achieves the accuracy of $53.1 \%$ and cannot beat the dominant option rule. We release the code and data of this paper for future research. ${ }^{23}$
\end{abstract}

\section{Introduction}

The research of question answering (QA), where a system needs to understand a piece of reading material and answer corresponding questions, has drawn considerable attention in recent years. While various QA datasets have been constructed to study how a QA system can understand a specific passage, the common sense knowledge and so on (Rajpurkar et al., 2016; Lai et al., 2017; Dunn et al., 2017; Rajpurkar et al., 2018), most questions in these datasets could be given their answers by extracting from a few relevant sentences so that the model only needs to find a small set of supporting evidences, whose temporal ordering does not effect the final answer. In other words, these questions are raised only considering a fixed document. However, in the real-life question answering, a question could have its timelines. To infer the answer, a good model needs to understand series of timeline information. For example, the question "how many points did Lebron James have?" would have different answers based on the time when the question was asked during a basketball game, and the answer would continuously change during the game. The other question "Which team would first earn 10 points?" would require a system to track down information of scoring points along the timeline until one team achieves 10 points.

According to the analysis above, we consider the timeline-based question answering problem as a gap which has not been covered by existing datasets. Thus, in this work we hope to construct a dataset where passages and questions both have timelines and question respondents are required to judge what information should be gathered for the questions involved in a timeline. Such a timeline inferenceinvolved QA dataset introduces a new research line of reading comprehension, that evaluates the ability of understanding temporal information of a QA model.

Additionally, the real-world questions are often involved in some math calculation, such as addition, subtraction and counting. To answer the questions correctly, one not only needs to locate some specific sentences, but also do calculation or comparison on the extracted evidence. For example, "How many points did the winner team win?" needs one system to perform subtraction on the final score to get the correct answer.

\footnotetext{
* This denotes equal contribution.

${ }^{1}$ https://nba.hupu.com/games

${ }^{2}$ code: https://github.com/PKU-TANGENT/GAReader-LiveQA

${ }^{3}$ data: https://github.com/PKU-TANGENT/LiveQA
} 
1: 第一节两队得分之和能否达到 51 分或更多?

Will the sum of the scores of the two teams

in the first quarter reach 51 points or more?

2: 本场勒布朗-詹姆斯能否得到 35 分或更多?

Will Lebron James get 35 points or more in

this match?

3: 暂停回来, 开拓者首次进攻会不会得分?

Will the Portland Trailblazers score in the

first attack after the timeout ends?

4: 本节比赛双方还能否再次命中三分球?

Will another three-pointer take place in the rest of the quarter?

Figure 1: Question Examples from the LiveQA dataset.

To these ends, we construct a QA dataset LiveQA based on a Hupu-live-broadcasting-dataset, which is a set of Chinese live-broadcasting passages of NBA. Hupu is a sports news website that has livebroadcasting for basketball games. In the Hupu-live-broadcasting, the host of one sport game describes the details of the game vividly with emotion and different sentence structures, and presents many gamerelated quizzes during the game. We collect the description texts and their quizzes into LiveQA. Answering the quizzes requires one model to correctly understand the timeline information of the context: some quizzes ask about information of one-whole quarter of the game or which player reaches a certain score earlier. Thus, the model needs to fully understand the temporal information of the live-broadcasting and then performs inference based on the temporal information. Figure 1 shows four question examples in the LiveQA dataset. Answering the first two questions requires an addition math operation, and the $3^{\text {rd }}$ and $4^{\text {th }}$ questions need comparison operation. Meanwhile, we can see that all these questions are time-dependent and require temporal inference.

In summarize, the main characteristics of our LiveQA dataset include the following two aspects. Firstly, the questions are time-awared. The model needs temporal inference to obtain the final answer. Secondly, in our dataset, reading comprehension is not limited to extracting a few specific text spans from the document, but is involved with math calculation. These characteristics make LiveQA challenging for previous QA systems to answer its questions. In this paper, we present an analysis of the resulting dataset to show how these characteristics appear in the data. We also show how questions are involved with temporal inference, and these questions also require mathematical inference. To demonstrate how these characteristics affect the performance of the QA model, we design a pipeline method, which first tries to find supporting sentences and then uses a strong baseline multi-hop inference model named Gated-Attention Reader, to judge the baseline performance on LiveQA. Our experimental results show that such strong baseline model only slightly exceeds random choice, which achieve $53.1 \%$ and cannot beat the dominant option rule. The analysis and experimental results show how this dataset can effectively examine how a QA system can perform multi-hop temporal and mathematical inference, which is not covered by previous studies.

The following of this paper is organized as follows: In section 2, we give a brief introduction of current QA research lines and research on live text processing. In section 3, we describe how we constructed the dataset. In section 4, we give statistics of the dataset and analyse the timelineness and mathematical 


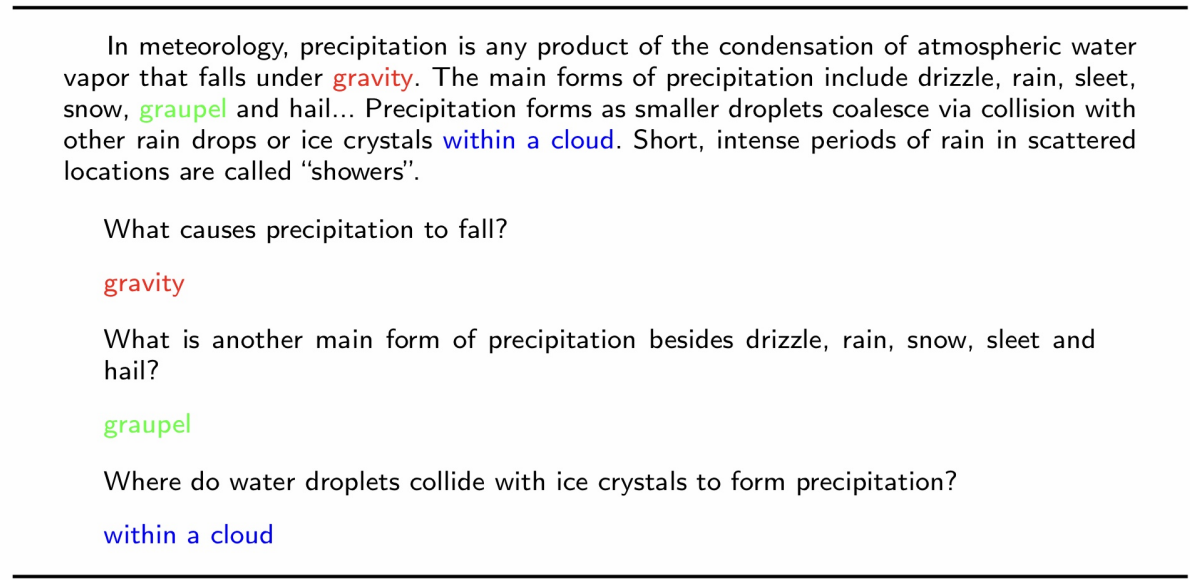

Figure 2: Examples of question-answer pairs in SQuAD

inference in the data. In section 5, we give evaluation results of baseline models and error analysis.

\section{Related Works}

In this section, we mainly introduce the various QA datasets which can be categorized as datasets with extractive answers, datasets with descriptive answers and datasets with multiple-choice questions.

\subsection{Datasets with Extractive Answers}

A number of QA datasets consist of numerous documents or passages which have considerable length. Each passage is equipped with several questions, answers of which are segments of the passage. The goal of a reading comprehension model is to find the correct text span. In other words, it may offer a begin position and an end position in the passage instead of generating the words itself. Such corpora are regarded as datasets with extractive answers.

The most famous dataset of this kind is Stanford Qustion Answering Dataset (SQuAD) (Rajpurkar et al., 2016). SQuAD v1.0 consists of 107,785 question-answer pairs compiled by crowdworkers from 536 Wikipedia articles, and is much larger than previous manually labeled datasets. Over 50,000 unanswerable questions are added in SQuAD v2.0 (Rajpurkar et al., 2018). It is more challenging for existing models because they have to make more unreliable guesses. As performances on SQuAD have become a common way to evaluate models, some experts regard SQuAD as the ImageNet (Deng et al., 2009) dataset in the NLP field.

Another frequently used dataset with extractive answers is CNN/Daily Mail dataset (Hermann et al., 2015), which was released by Google DeepMind and University of Oxford in 2015. One shining point of it is that each entity is anonymised by using an abstract entity marker to prevent models from using word-level information or n-gram models to find the answer rather than comprehending the passage.

CBT (Hill et al., 2015a), NewsQA (Trischler et al., 2016), TriviaQA (Joshi et al., 2017) and many other datasets can also be categorized into this class. They constitute a high proportion of MRC datasets, and can test the abilities of extractive models in various ways. The most closest work to ours is DROP (Dua et al., 2019), which is a QA dataset that requires discrete reasoning over the content of paragraphs. It requires the system to extract various pieces of numerical evidence and perform calculation on top of the numbers. Thus, we aim to construct a novel dataset, on which extractive models are likely to make mistakes in looking for the location of an answer, that the dataset can open a new research line for question answering by testifying the ability of models to understand timelineness.

\subsection{Datasets with Descriptive Answers}

Instead of selecting a span from the passage, datasets with descriptive answers require a reading comprehension model to generate whole and stand-alone sentences. These corpora are more closer to reality, 


\begin{tabular}{|c|c|}
\hline Original Version & Anonymised Version \\
\hline $\begin{array}{l}\text { Context } \\
\text { The BBC producer allegedly struck by Jeremy } \\
\text { Clarkson will not press charges against the "Top } \\
\text { Gear" host, his lawyer said Friday. Clarkson, who } \\
\text { hosted one of the most-watched television shows } \\
\text { in the world, was dropped by the BBC Wednesday } \\
\text { after an internal investigation by the British broad- } \\
\text { caster found he had subjected producer Oisin Tymon } \\
\text { "to an unprovoked physical and verbal attack."... }\end{array}$ & $\begin{array}{l}\text { the ent } 381 \text { producer allegedly struck by ent } 212 \text { will } \\
\text { not press charges against the " ent } 153 \text { " host, his } \\
\text { lawyer said friday . ent } 212 \text {, who hosted one of the } \\
\text { most - watched television shows in the world, was } \\
\text { dropped by the ent } 381 \text { wednesday after an internal } \\
\text { investigation by the ent } 180 \text { broadcaster found he } \\
\text { had subjected producer ent } 193 \text { " to an unprovoked } \\
\text { physical and verbal attack." ... }\end{array}$ \\
\hline $\begin{array}{l}\text { Query } \\
\text { Producer } \mathbf{X} \text { will not press charges against Jeremy } \\
\text { Clarkson, his lawyer says. }\end{array}$ & $\begin{array}{l}\text { producer } \mathbf{X} \text { will not press charges against ent } 212 \text {, } \\
\text { his lawyer says . }\end{array}$ \\
\hline $\begin{array}{l}\text { Answer } \\
\quad \text { Oisin Tymon }\end{array}$ & ent 193 \\
\hline
\end{tabular}

Figure 3: An example of anonymised entity in CNN/Daily Mail

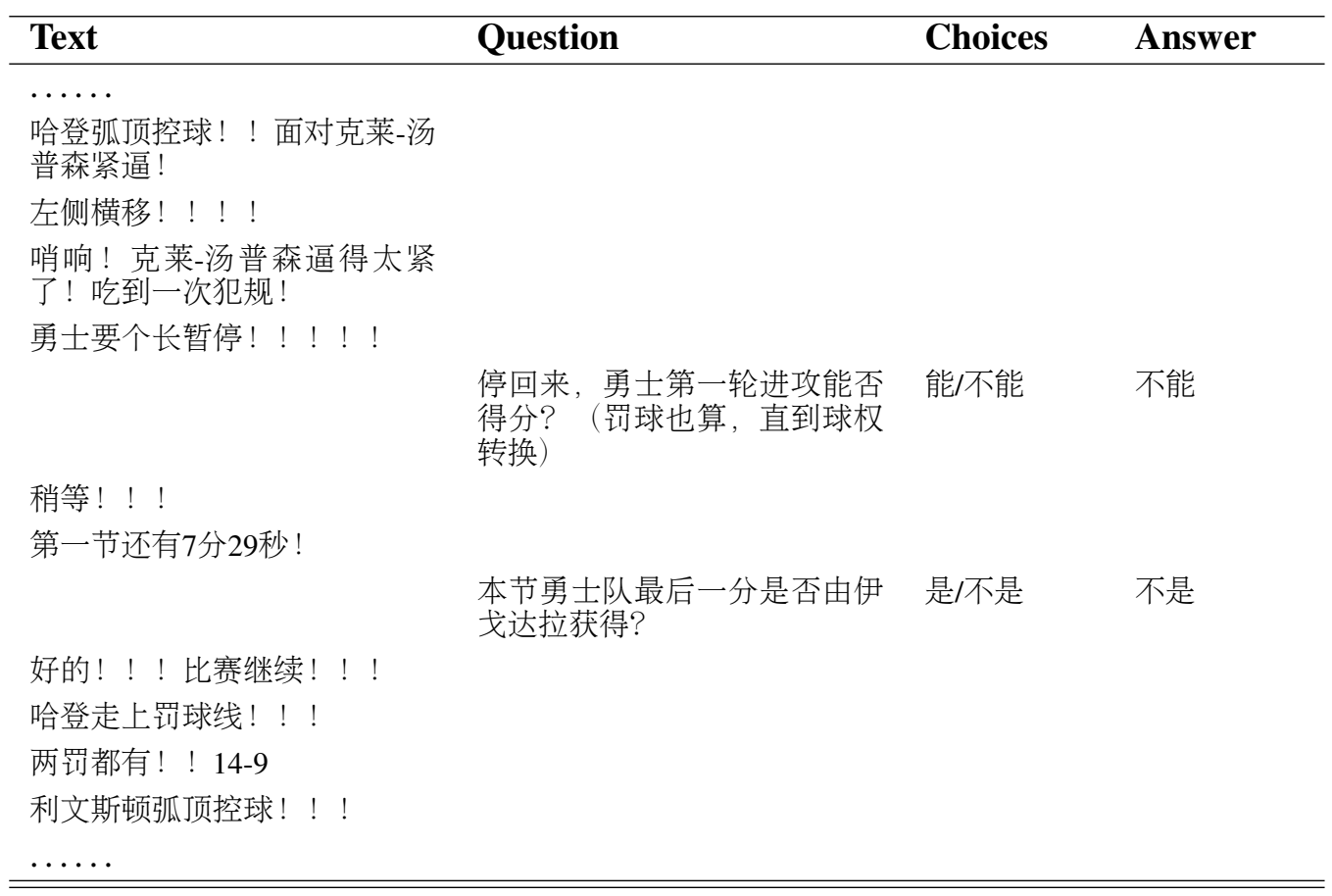

Figure 4: A partial example of LiveQA timeline.

because most questions in the real world cannot be solved simply by presenting a span or an entity. This kind of dataset is getting popular nowadays, and may be the trend of the development of MRC datasets. MS MARCO (Microsoft MAchine Reading Comprehension) (Nguyen et al., 2016) is a dataset released by Microsoft in 2016. This dataset aims to address the questions and documents in the real world, as its questions are sampled from Bing's search query logs and its passages are extracted from web documents retrieved by Bing. The questions in MS MARCO are about ten times as many as SQuAD, and each question is equipped with a human generated answer. The dataset also includes unanswaerable questions. All of the above characteristics make MS MARCO worthy of trying.

NarrariveQA (Kočiský et al., 2017) is another dataset with descriptive answers released by DeepMind and University of Oxford in 2017. The dataset consists of stories, books and movie scripts, with human written questions and answers based solely on human-generated abstractive summaries. Answering such questions requires readers to integrate information which may distribute across several statements throughout the document, and generate a cogent answer on the basis of this integrated information. In other words, they test that the reader comprehends language, not just that it can pattern match. We judge it a referential advantage of a dataset, so LiveQA requires the ability of tracking events as well as we 
show in Figure 4, which will be detailedly introduced in following sections.

\subsection{Datasets with Multiple-choice Questions}

Datasets with descriptive answers have various advantages, but they are relatively difficult to evaluate the system performance precisely and objectively. Thus, corpuses with more gradable QA-pairs are also needed, which leads to the development of datasets with multiple-choice questions. Through diversified types of questions, these datasets can examine almost every ability of a reading comprehension model mentioned above and are easier to get a conclusive score. Many datasets of this kind have been released in recent years, and they have covered multiple domains. For example, RACE (Lai et al., 2017) and CLOTH (Xie et al., 2017) are collected from English exams, MCTest (Richardson et al., 2013) is sampled from friction stories, and ARC (Clark et al., 2018) is extracted from science-related sentences. However, there is still not a reliable dataset which is built on sports events for MRC. Thus, our LiveQA dataset has the potential for filling several gaps in the field of MRC.

\subsection{Live Text Processing}

Previously, various studies have been conducted on automatically generate sports news from live text commentary scripts, which has been seen as a summarization task. Zhang et al. (2016) proposed an investigation on summarization of sports news from live text commentary scripts, where they treat this task as a special kind of document summarization based on sentence extraction in a supervised learning to rank framework. Yao et al. (2017) further verify the feasibility of a more challenging setting to generate news report on the fly by treating live text input as a stream for sentence selection. Wan et el. (2016) studied dealing with the summarization task in Chinese. All these studies focuses on using the live text commentary scripts as the input of summarization and selecting sentences to form the summary. So far, we are the first to point out the importance of timelineness and mathematical reasoning in understanding live text commentary scripts.

\section{LiveQA: Dataset Construction}

In this section we introduce how to construct LiveQA from the raw Hupu text and present the corpus statistics. The whole process of building LiveQA mainly includes crawling the raw data and acquiring the game texts with corresponding quizzes.

\subsection{Data Crawling}

In Hupu, each game has a unique ID which is connected with its url. We collected the IDs from the Hupu's live schedule pages. Their formats are https://nba.hupu.com/games/year-month-date. There are links to all the NBA games so that their IDs can be saved. After we saved the IDs into a file, we used the web debugging tool Fiddler to get a sample of the url of a game, and then changed the IDs in the url to make access to all the games. We are authorized by the legal department of hupu website to construct the dataset for only academical purpose.

\subsection{Data Processing}

Most previous datasets usually do not care for the storing positions of the passages and their questions. But in our dataset, the quizzes and the contexts shouldn't be separated because the time (the position) one quiz occurs is quite important for the final answer. If we separate the quizzes and their contexts, most quizzes may have different answers and cannot be answered even by human. Here we use some rules to clean the dataset. The lines starting with '@' are always interactions between the host and some active readers, which are irrelevant to the game. During the half-time break, the host will give out some "gift" questions to please the readers waiting for the second-half. Some of the questions appear like normal quizzes, but they need information outside the game to answer them, thus we exclude them from the data (i.e.. which team won more matches in the history?). Usually they have a prefix - "中场福利” in common. Besides, we exclude the descriptions of pictures from our data. 


\subsection{Data Structure}

Here we give an explanation for the structure for each independent data sample.

For each live-stream of one match, the timeline data is sorted in time order, where the questions are inserted into the corresponding timeline position so that the timeline features of the questions could be inferred. As we show in Figure 4, the plain content text and the question text share the same timeline, but question records have choices and answers along with the text. For each record in the timeline, it either contains a piece of live-stream text or a question bonded with the corresponding choices and the correct answer. Each question has two answer choices.

\section{Dataset Statistics}

\begin{tabular}{ll}
\hline Element & Count \\
\hline Document & 1,670 \\
Sentences in Total & 1786616 \\
Sentences in Average & 1069.83 \\
Quizzes in Total & 117050 \\
Quizzes in Average & 70.09 \\
\hline
\end{tabular}

Table 1: The details of statistics of the dataset.

We show the statistics of the dataset in Table 1. The LiveQA dataset contains 1,670 documents, each of which has 70.09 quizzes and 1069.83 sentences on average. Next we analyze the questions from two different views. First, we simply classify the questions according to the positions of their answers. In general, some of the questions can be solved by extracting information from neighboring sentences, which involves a time period of the origin game. Such questions occupy $68.6 \%$ of all the questions. Some questions can be replied only by summarizing all the information after the game ends and occupy about $30.6 \%$. Still, there exists a small percentage $(0.8 \%)$ of questions which are impossible to be answered from the passage. Table 2 lists some examples for each type of questions.

Because most of the questions are associated with some numerical data in the game, we also classify the questions according to how the numerical data is performed. Four types of operations are commonly used including: Comparison, Calculation, Inference and Tracking. Then the questions are correspondingly classified are introduced in the following subsections. We also give some examples in Table 3.

\subsection{Comparison}

To answer the comparison questions, we usually need to find the comparative figures for the corresponding objects. For example, the commentator asks which of the two players will score more or which team will win. The second row in Table 3 belong to the Comparison questions. The easiest way to solve this kind of questions is to find the two figures appearing in the text and comparing them. It is likely to acquire such figures after the game ends, and the specific figures usually appear together in a summary of the game in the end. Thus, matching techniques are still necessary to the final answer.

\subsection{Calculation}

The Calculation questions require extracting two or three figures and calculating their sum or difference. They differ from the Comparison questions in two ways - the figures are more scattered and a calculation step is needed. This means that a respondent has to look for more information efficiently. After the figures are obtained, if a respondent misjudges the type or the direction of the calculation, he will still probably get a wrong answer. Similar to the Comparison questions, the Calculation questions are mainly dependent on the correct sentences where the figures are located. These two kinds of questions are relatively easy compared to those ones which are not based on certain sentences. The second row in Figure 3 give two example questions. 


\begin{tabular}{|c|c|c|c|}
\hline $\begin{array}{l}\text { Question } \\
\text { type }\end{array}$ & Proportion & Example & Translation \\
\hline \multirow[t]{2}{*}{$\begin{array}{l}\text { Answered } \\
\text { after the } \\
\text { game ends }\end{array}$} & \multirow[t]{2}{*}{$30.6 \%$} & $\begin{array}{l}\text { 本场森林狼能否赢快 } \\
\text { 船4分或更多? }\end{array}$ & $\begin{array}{l}\text { Will the Timberwolves beat } \\
\text { the Clippers by more than } 4 \\
\text { points? }\end{array}$ \\
\hline & & 本场比赛谁会赢? & Which team will win? \\
\hline \multirow[t]{2}{*}{$\begin{array}{l}\text { Answered } \\
\text { through the } \\
\text { context }\end{array}$} & \multirow[t]{2}{*}{$68.6 \%$} & $\begin{array}{l}\text { 第二节谁先命中三分 } \\
\text { 球? }\end{array}$ & $\begin{array}{l}\text { Which team will make a } \\
\text { three-pointer first in the sec- } \\
\text { ond quarter? }\end{array}$ \\
\hline & & $\begin{array}{l}\text { 首节最后一分会不会由 } \\
\text { 罚球获得? }\end{array}$ & $\begin{array}{l}\text { Will the last point in the first } \\
\text { quarter scored through a free- } \\
\text { throw? }\end{array}$ \\
\hline \multirow[t]{2}{*}{$\begin{array}{l}\text { Impossible } \\
\text { to answer }\end{array}$} & \multirow[t]{2}{*}{$0.8 \%$} & $\begin{array}{l}\text { 第 二节 比 赛 开 } \\
\text { 始 } 1 \text { 分 } 30 \text { 秒 时 间 内 会 } \\
\text { 不会有三分球命中? }\end{array}$ & $\begin{array}{l}\text { Will a three-pointer be made } \\
\text { in the first } 90 \text { s of the second } \\
\text { quarter? }\end{array}$ \\
\hline & & $\begin{array}{l}\text { 本场比赛会不会在北 } \\
\text { 京时间 } 10 \text { 时 } 58 \text { 分之前结 } \\
\text { 束? }\end{array}$ & $\begin{array}{l}\text { Will the game end before } \\
10: 58 \text { a.m.? }\end{array}$ \\
\hline
\end{tabular}

Table 2: Questions statistics and examples sorted by the location of their corresponding evidence.

\begin{tabular}{|c|c|c|c|}
\hline $\begin{array}{l}\text { Question } \\
\text { type }\end{array}$ & Proportion & Example & Translation \\
\hline \multirow[t]{2}{*}{ Comparison } & \multirow[t]{2}{*}{$16.6 \%$} & $\begin{array}{l}\text { 勒布朗-詹姆斯本场能否 } \\
\text { 得到26分或更多? }\end{array}$ & $\begin{array}{l}\text { Will Lebron James get } 26 \\
\text { points or more in this game? }\end{array}$ \\
\hline & & 本场谁的得分会更高？ & $\begin{array}{l}\text { Who will get higher score in } \\
\text { this game? }\end{array}$ \\
\hline \multirow{2}{*}{ Calculation } & \multirow{2}{*}{$25.4 \%$} & $\begin{array}{l}\text { 本场凯尔特人能否赢猛 } \\
\text { 龙3分或更多? }\end{array}$ & $\begin{array}{l}\text { Will the Celtics beat the Rap- } \\
\text { tors by more than } 3 \text { points? }\end{array}$ \\
\hline & & $\begin{array}{l}\text { 本场两队总得分能否达 } \\
\text { 到207分或更多? }\end{array}$ & $\begin{array}{l}\text { Will the total score of the } \\
\text { two teams reach } 207 \text { points } \\
\text { or more? }\end{array}$ \\
\hline \multirow[t]{2}{*}{ Inference } & \multirow[t]{2}{*}{$28.5 \%$} & $\begin{array}{l}\text { 暂停回来, 雷霆队首次 } \\
\text { 进攻能否得分? }\end{array}$ & $\begin{array}{l}\text { After the timeout, will the } \\
\text { Thunder score in their first } \\
\text { round of attack? }\end{array}$ \\
\hline & & $\begin{array}{l}\text { 第二节比赛雷霆队最后 } \\
\text { 一分会不会由威斯布鲁 } \\
\text { 克得到? }\end{array}$ & $\begin{array}{l}\text { Will the last point of the } \\
\text { Thunder in the second quar- } \\
\text { ter be got by Westbrook? }\end{array}$ \\
\hline \multirow[t]{2}{*}{ Tracking } & \multirow[t]{2}{*}{$29.5 \%$} & $\begin{array}{l}\text { 太阳队能否在本场命 } \\
\text { 中8个或更多三分球? }\end{array}$ & $\begin{array}{l}\text { Will the Suns make } 8 \text { three- } \\
\text { pointers or more in this } \\
\text { game? }\end{array}$ \\
\hline & & $\begin{array}{l}\text { 凯文-乐福首节犯规数会 } \\
\text { 不会达到2次? }\end{array}$ & $\begin{array}{l}\text { Will Kevin Love commit } 2 \\
\text { fouls or more in the first quar- } \\
\text { ter? }\end{array}$ \\
\hline
\end{tabular}

Table 3: Questions statistics and examples sorted by how the inference process is done.

\subsection{Inference}

The third and fourth type of questions require the ability of summarizing and tracking information. A question of the third type needs a respondent to infer some figures through the text. For example, a question may be "After this timeout, will the Cavaliers score in the first round of attack?". The commentator 
obviously will not say that "The Cavaliers scored 2 points." or "The Cavaliers didn't score." A respondent may get the answer as "JR Smith makes a 2-point shot." Another example is "Will the last point of this quarter be scored through a free throw?" The information comes from the text of "Anthony Davis makes his second free throw ... The match ends!". It is impossible to get a reasonable answer by matching.

\subsection{Tracking}

The Tracking questions require more scattered information. A respondent should collect and accumulate specific information from a part of the passage, as the question is based on events happening repeatedly in a quarter or half of the game. For example, some questions ask about how many free-throws a player $A$ will make in a quarter. As this figure does not appear in the passage, a respondent needs to count how many times the event ' $A$ makes a free-throw' occurs. In other words, it is necessary to track events relevant to the player ' $A$ ' and 'free-throw'. When the player $(A)$ is replaced with one team name, the new question is even more difficult because the information about each player belonging to the team should be tracked. Therefore, information tracking leads this kind of questions to be the most challenging ones in the dataset.

\section{Baseline Models and Results}

\subsection{Models}

To evaluate the QA performance on the LiveQA dataset, we implement 3 baseline models. The first is based on random selection, where the system randomly chooses a choice as the answer. The second is to choose the dominant option of each question. More concretely, $80.0 \%$ of questions are in format of 'yes' and 'no', where $57.8 \%$ has the answer 'no'. For the other multiple choice questions, $50.6 \%$ of them take the second option as the right answer. Thus, for 'yes/no' questions, we choose 'no', otherwise we choose the second option.

We also build a neural-network style baseline for our dataset to evaluate how state-of-the-art QA systems perform on the LiveQA dataset. Due to the uniqueness of our dataset, most of existing machine comprehension models are not suitable to it. For example, the QANet (Yu et al., 2018) model, which used to be a state-of-art model of SQuAD (Rajpurkar et al., 2016), is unavailable because it predicts the probability distribution of an answer's starting position and ending position in the context. But in LiveQA, a number of right answers do not directly appear in the context (e.g. an answer in format of 'can' or 'cannot'). Up to now, none of machine reading comprehension models has been designed for a dataset with consideration of timeline and mathematical computations. That means that the existing ones will not be likely to perform well on our dataset. The closest work to ours is multi-hop question answering, and thus we use a novel model Gated-Attention Reader (Dhingra et al., 2016) to experiment on LiveQA.

Gated-Attention Reader (GA) is an attention mechanism which uses multiplicative interactions between the query embedding and intermediate states of a recurrent neural network reader. GA enables a model to scan one document and the questions iteratively for multiple passes, and thus the multi-hop structure can target on most relevant parts of the document. It used to be the state-of-art model of several datasets, such as CNN/Daily Mail dataset (Hermann et al., 2015) and CBT dataset (Hill et al., 2015b).

The full context, which is usually composed of more than 1,000 sentences on average, is too heavy for GA as input. To apply GA to our dataset, we propose a pipeline method to first extract a set of candidate evidence sentences from the full content, and then apply the GA model on this set of sentences to predict the final answer. We employ TF-IDF style matching score to extract 50 most relevant sentences as the supporting evidence. To improve the accuracy of selecting the evidence candidates, if the question clearly requires some information after the game ends, we use the ending part of the content as the input.

Specifically, taken the embedding representation of a token, the Bi-directional Gated Recurrent Units (BiGRU) process the sequence in both forward and backward directions to produce two sequences of token-level representations, which are concatenated at the output as the final representation of the token. To perform multi-hop inference, the GA model reads the document and the query over $k$ horizontal layers, where layer $k$ receives the contextual embeddings $X_{(k-1)}$ of the document from the previous layer. 
At each layer, the document representation $D^{(k)}$ is computed by taking the full output of a document BiGRU where the previous layer embedding $X_{(k-1)}$ is the input. At the same time, a layer-specific query representation $Q^{(k)}$ is computed as the full output of a separate query BiGRU taking the query embedding $Y$ as the input. The Gated-Attention is applied to $D^{(k)}$ and $Q^{(k)}$ to compute the contextual embedding $X^{(k)}$.

$$
X^{(k)}=\operatorname{GAttn}\left(\operatorname{BiGRU}\left(X^{(k-1)}\right), \operatorname{BiGRU}(Y)\right)
$$

After obtaining the query-awared document representation, we perform answer prediction by matching the similarity of answer and content. We use bidirectional Gated Recurrent Units to encode the candidate answers into vectors $\left.A^{(} i\right)$, and then we compute matching score between summarized document and candidates using a bilinear attention. Finally we calculate the probability distribution of the options with softmax. The operations are similar to those in RACE (Lai et al., 2017).

$$
s=\operatorname{softmax}\left(\left[\operatorname{Blin}\left(A^{i}, D^{(k)}\right) ;\right]_{n}^{i=1}\right)
$$

\subsection{Model Evaluation}

\begin{tabular}{ll}
\hline Model & Acc \\
\hline Random & $50.0 \%$ \\
Dominant & $\mathbf{5 6 . 4 \%}$ \\
GA & $53.1 \%$ \\
\hline
\end{tabular}

Table 4: The results of different baseline models on the test set. Random denotes randomly selecting an answer. Dominate denotes selecting the dominate option. GA denotes the gated-attention reader.

For the three baseline models, performance is reported with the accuracy on the test set in Table 4 . The random selection method (Random) scores 50.0\%, while the dominant option method (Dominate) reaches a score of $56.4 \%$, which shows that our dataset does not have a certain pattern for the answers. Meanwhile, GA, which is a strong baseline for previous question answering problems, failed to perform better than the dominant option method and only achieves a score of $53.1 \%$. Such results show that our dataset is challenging and needs further investigation for model design. In future work, how to incorporate temporal information and mathematical calculation into a QA model is the focus.

\subsection{Case Study}

In this subsection, we further analyze the prediction ability of the GA model. Table 5 shows some prediction cases in experimental results. From the first two questions, we can see that the model gives the correct answers when judging the result of a specific event. But for the other three questions which involve multiple events, the model fails to answer them correctly. A possible explanation is that, although GA is designed for multi-hop inference, it lacks ability in both information tracking and math calculation, which makes it difficult for the model to track down some complicated events.

We can see, for reading comprehension models that extract answers based on the similarity between the answer and the content, they would fail on LiveQA due to the fact that they cannot track down temporal information nor perform mathematical calculation. To outperform existing models on LiveQA, the system should consider focusing on tracking information of a certain event through the timeline. It should also have the ability to perform mathematical inference between different contents.

\section{Conclusion}

In this paper, we present LiveQA, a question answering dataset constructed from play-by-play live broadcast. LiveQA can evaluate a machine reading comprehension model in its ability to understand the timeline, track events and do mathematical calculation. It consists of $117 \mathrm{k}$ questions, which are timedependent and need math inference. Due to the novel characteristics, it is hard for existing QA models 


\begin{tabular}{|c|c|c|c|}
\hline Question & Translation & Correct answer & $\begin{array}{l}\text { Answer given } \\
\text { by the model }\end{array}$ \\
\hline $\begin{array}{l}\text { 跳球之争! 本场比赛 } \\
\text { 哪支球队获得第一轮 } \\
\text { 进攻球权? }\end{array}$ & $\begin{array}{l}\text { Jump ball fight! } \\
\text { Which team will win } \\
\text { the chance of the first } \\
\text { round of offence? }\end{array}$ & $\begin{array}{l}\text { 勇士(The War- } \\
\text { riors) }\end{array}$ & $\begin{array}{l}\text { 勇士(The War- } \\
\text { riors) }\end{array}$ \\
\hline 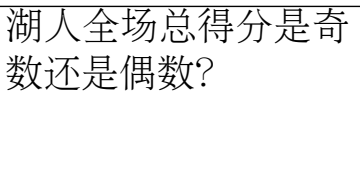 & $\begin{array}{l}\text { Will the total score of } \\
\text { the Lakers at the end } \\
\text { of the game be odd or } \\
\text { even? }\end{array}$ & 奇数(odd) & 奇数(odd) \\
\hline $\begin{array}{l}\text { 尼克杨第二节能否命 } \\
\text { 中3分球? }\end{array}$ & $\begin{array}{l}\text { Can Nick Young make } \\
\text { a three pointer in the } \\
\text { second quarter? }\end{array}$ & 能(Yes) & 不能(No) \\
\hline $\begin{array}{l}\text { 第三节结束, } 76 \text { 人能 } \\
\text { 否领先湖人 } 4 \text { 分或更 } \\
\text { 多? }\end{array}$ & $\begin{array}{l}\text { At the end of the third } \\
\text { quarter, Will the } 76 \mathrm{ers} \\
\text { lead the Lakers by } 4 \\
\text { points of more? }\end{array}$ & 不能(No) & 能(Yes) \\
\hline 谁先获得30分? & $\begin{array}{l}\text { Who will score his } \\
\text { 30th point earlier? }\end{array}$ & $\begin{array}{l}\text { 24分 的 哈 } \\
\text { 登(James } \\
\text { Harden who has } \\
\text { got } 24 \text { points) }\end{array}$ & $\begin{array}{lr}25 \text { 分 的 } \text { 托 } \\
\text { 马 斯(Isaiah } \\
\text { Thomas who } \\
\text { has got } 25 \\
\text { points) }\end{array}$ \\
\hline
\end{tabular}

Table 5: Cases in the experimental results

to perform well on LiveQA. We expect our dataset will stimulate the development of more advanced machine comprehension models.

\section{Acknowledgement}

We thank the anonymous reviewers for their helpful comments on this paper. This work was partially supported by National Natural Science Foundation Project of China (61876009), National Key Research and Development Project (2019YFB1704002), and National Social Science Foundation Project of China (18ZDA295). The corresponding author of this paper is Sujian Li.

\section{References}

Peter Clark, Isaac Cowhey, Oren Etzioni, Tushar Khot, Ashish Sabharwal, Carissa Schoenick, and Oyvind Tafjord. 2018. Think you have solved question answering? try arc, the ai2 reasoning challenge. arXiv preprint arXiv:1803.05457.

Jia Deng, Wei Dong, Richard Socher, Li Jia Li, Kai Li, and Fei Fei Li. 2009. Imagenet: A large-scale hierarchical image database. In IEEE Conference on Computer Vision \& Pattern Recognition.

Bhuwan Dhingra, Hanxiao Liu, Zhilin Yang, William W Cohen, and Ruslan Salakhutdinov. 2016. Gated-attention readers for text comprehension. arXiv preprint arXiv:1606.01549.

Dheeru Dua, Yizhong Wang, Pradeep Dasigi, Gabriel Stanovsky, Sameer Singh, and Matt Gardner. 2019. Drop: A reading comprehension benchmark requiring discrete reasoning over paragraphs. In Proceedings of the 2019 Conference of the North American Chapter of the Association for Computational Linguistics: Human Language Technologies, Volume 1 (Long and Short Papers), pages 2368-2378.

Matthew Dunn, Levent Sagun, Mike Higgins, V Ugur Guney, Volkan Cirik, and Kyunghyun Cho. 2017. Searchqa: A new q\&a dataset augmented with context from a search engine. arXiv preprint arXiv:1704.05179. 
Karl Moritz Hermann, Tomas Kocisky, Edward Grefenstette, Lasse Espeholt, Will Kay, Mustafa Suleyman, and Phil Blunsom. 2015. Teaching machines to read and comprehend. In Advances in Neural Information Processing Systems, pages 1693-1701.

Felix Hill, Antoine Bordes, Sumit Chopra, and Jason Weston. 2015a. The goldilocks principle: Reading children's books with explicit memory representations. Computer Science.

Felix Hill, Antoine Bordes, Sumit Chopra, and Jason Weston. 2015b. The goldilocks principle: Reading children's books with explicit memory representations. arXiv preprint arXiv:1511.02301.

Mandar Joshi, Eunsol Choi, Daniel S. Weld, and Luke Zettlemoyer. 2017. Triviaqa: A large scale distantly supervised challenge dataset for reading comprehension.

Tomáš Kočiský, Jonathan Schwarz, Phil Blunsom, Chris Dyer, and Edward Grefenstette. 2017. The narrativeqa reading comprehension challenge.

Guokun Lai, Qizhe Xie, Hanxiao Liu, Yiming Yang, and Eduard Hovy. 2017. Race: Large-scale reading comprehension dataset from examinations. arXiv preprint arXiv:1704.04683.

Tri Nguyen, Mir Rosenberg, Song Xia, Jianfeng Gao, and Deng Li. 2016. Ms marco: A human generated machine reading comprehension dataset.

Pranav Rajpurkar, Jian Zhang, Konstantin Lopyrev, and Percy Liang. 2016. Squad: 100,000+ questions for machine comprehension of text. arXiv preprint arXiv:1606.05250.

Pranav Rajpurkar, Robin Jia, and Percy Liang. 2018. Know what you don't know: Unanswerable questions for squad. arXiv preprint arXiv:1806.03822.

Matthew Richardson, Christopher JC Burges, and Erin Renshaw. 2013. Mctest: A challenge dataset for the open-domain machine comprehension of text. In Proceedings of the 2013 Conference on Empirical Methods in Natural Language Processing, pages 193-203.

Adam Trischler, Wang Tong, Xingdi Yuan, Justin Harris, and Kaheer Suleman. 2016. Newsqa: A machine comprehension dataset.

Xiaojun Wan, Jianmin Zhang, Jin-ge Yao, and Tianming Wang. 2016. Overview of the nlpcc-iccpol 2016 shared task: Sports news generation from live webcast scripts. In Chin-Yew Lin, Nianwen Xue, Dongyan Zhao, Xuanjing Huang, and Yansong Feng, editors, Natural Language Understanding and Intelligent Applications, pages 870-875, Cham. Springer International Publishing.

Qizhe Xie, Guokun Lai, Zihang Dai, and Eduard Hovy. 2017. Large-scale cloze test dataset created by teachers. arXiv preprint arXiv:1711.03225.

Jin-ge Yao, Jianmin Zhang, Xiaojun Wan, and Jianguo Xiao. 2017. Content selection for real-time sports news construction from commentary texts. In Proceedings of the 10th International Conference on Natural Language Generation, pages 31-40.

Adams Wei Yu, David Dohan, Minh-Thang Luong, Rui Zhao, Kai Chen, Mohammad Norouzi, and Quoc V Le. 2018. Qanet: Combining local convolution with global self-attention for reading comprehension. arXiv preprint arXiv:1804.09541.

Jianmin Zhang, Jin-ge Yao, and Xiaojun Wan. 2016. Towards constructing sports news from live text commentary. In Proceedings of the 54th Annual Meeting of the Association for Computational Linguistics (Volume 1: Long Papers), pages 1361-1371. 